\title{
ON EVENTUAL STABILITY OF IMPULSIVE SYSTEMS OF DIFFERENTIAL EQUATIONS
}

\author{
A. A. SOLIMAN
}

(Received 8 August 2000)

\begin{abstract}
The notions of Lipschitz stability of impulsive systems of differential equations are extended and the notions of eventual stability are introduced. New notions called eventual and eventual Lipschitz stability. We give some criteria and results.
\end{abstract}

2000 Mathematics Subject Classification. 34D20.

1. Introduction. The mathematical theory of impulsive systems of differential equations is much richer in problems in comparison with the corresponding theory of ordinary differential equations. That is why the impulsive systems of differential equations are adequate apparatus for the mathematical simulation of numerous processes and phenomena studied in biology, physics, technology, and so forth, such processes and phenomena are characterized by the fact that at certain moments of their evolution they undergo rapid changes that is why in their mathematical simulation it is convenient to neglect the duration of these changes and assume that such processes and phenomena change their state momentarily by jump. Recently the study of such systems has been very intensive (see the monographs by $[1,5,6]$, and the references therein).

Lipschitz stability notion of systems of ordinary differential equations are introduced by Dannan and Elaydi [2]; then Kulev and Baĭnov [3, 4] introduced these notions for impulsive systems of differential equations.

The purpose of this paper is to introduce the notions of eventual stability of impulsive systems of differential equations and the notions of Lipschitz stability of [4] are extended to a new type of stability of impulsive systems, namely, eventual Lipschitz stability. These notions lie somewhere between Lipschitz stability of [4], on one side and eventual stability on the other side.

The paper is organized as follows. In Section 1, introduction of preliminaries definitions and notions which will be used in this paper. In Section 2, we introduce the notion of eventual stability of impulsive systems. In Section 3, we extend the notion of eventual Lipschitz stability and give some criteria and some examples.

Consider the impulsive systems

$$
x^{\prime}=f(t, x),\left.\quad \Delta x\right|_{t=t_{k}}=I_{k}(x), \quad x\left(t_{0}+0\right)=x_{0},
$$

where $f: J \times \mathbb{R}^{n} \rightarrow \mathbb{R}^{n}, J=\left[t_{0}, \infty\right), I_{k}: \mathbb{R}^{n} \rightarrow \mathbb{R}^{n}, 0 \leq t_{0}<t_{1}<t_{2}<\cdots,\left.\Delta_{x}\right|_{t=t_{k}}=$ $x\left(t_{k}+0\right)-x\left(t_{k}-0\right), \mathbb{R}^{n}$ is the $n$-dimensional Euclidean space, and $\|x\|$ is any norm of the vector $x \in \mathbb{R}^{n}$. 
The impulsive system of the form (1.1) were described in detail by the authors in $[1,5,6]$. Let $x(t)=x\left(t, t_{0}, x_{0}\right)$ be a solution of (1.1) satisfying the initial condition $x\left(t_{0}+0\right)=x_{0}$, and which is defined on the interval $\left(t_{0}, \infty\right)$. Let $t=t_{k}, k=1,2, \ldots$ be the moments at which the integral curve of this solution meets the hypersurfaces. In further considerations, we use the notations

$$
s(\rho)=\left\{x \in \mathbb{R}^{n}:\|x\|<\rho, \rho>0\right\}, \quad\|A\|=\sup _{\|x\|<1}|A x|,
$$

where $A$ is an arbitrary $n \times n$ matrix.

$$
K=\left\{a \in C\left[\mathbb{R}^{+}, \mathbb{R}^{+}\right], a \text { is strictly increasing in } \mathbb{R}^{+}=[0, \infty), a(0)=0\right\} .
$$

Consider the impulsive variational systems of (1.1)

$$
\begin{gathered}
y^{\prime}=f_{x}(t, 0) ; \quad t \neq t_{k} ;\left.\quad \Delta y\right|_{t=t_{k}}=I_{k}^{\prime}(0) y ; \quad y\left(t_{0}+0\right)=y_{0}, \\
z^{\prime}=f_{x}\left(t, x\left(t, t_{0}, x_{0}\right)\right) z, \quad t \neq t_{k},\left.\quad \Delta z\right|_{t=t_{k}}=I_{k}^{\prime}\left(x\left(t_{k}, t_{0}, x_{0}\right)\right) z, \quad z\left(t_{0}+0\right)=z_{0} .
\end{gathered}
$$

Furthermore, we consider the linear impulsive system

$$
x^{\prime}=A(t) x, \quad t \neq t_{k} ;\left.\quad \Delta x\right|_{t=t_{k}}=B_{k} x ; \quad x\left(t_{0}+0\right)=x_{0},
$$

where $f_{x}=\partial f / \partial x, I_{k}^{\prime}(x)=\partial I_{k} / \partial x$ and $x\left(t, t_{0}, x_{0}\right)$ be any solution of (1.1) satisfying the initial condition $x\left(t, t_{0}, x_{0}\right)=x_{0}$, and $A$ is an $n \times n$ matrix defined in $J$, and $B_{k}$, $k=1,2, \ldots$ are constant $n \times n$ matrices.

The fundamental matrix solution $\Phi\left(t, t_{0}, x_{0}\right)$ of system (1.5) is defined by

$$
\Phi\left(t, t_{0}, x_{0}\right)=\frac{\partial x\left(t, t_{0}, x_{0}\right)}{\partial x_{0}}, \quad t \neq t_{k},
$$

(see [5, Theorem 2.4.1]).

Following $[1,5,6]$, if the fundamental matrix solution $u(t, s)$ of system (1.6) without impulses

$$
x^{\prime}=A(t) x .
$$

Then the fundamental matrix solution $w(t, s)$ of system (1.6) is defined by

$$
w(t, s)= \begin{cases}u(t, s), & t_{k-1}<s<t<t_{k}, \\ u\left(t, t_{k}\right)\left(E+B_{k}\right) u\left(t_{k}, s\right), & t_{k-1}<s<t_{k}<t \leq t_{k+1}, \\ u\left(s, t_{k+1}\right) \prod_{j=i}^{1}\left(E+B_{k+j}\right) u\left(t_{k+j}, t_{k+j-1}\right)\left(E+B_{k}\right) u\left(t_{k}, s\right), & t_{k-1}<s \leq t_{k}<t_{k}<t_{k+1}<t \leq t_{k+i+1},\end{cases}
$$

where $E$ is the unit $n \times n$ matrix.

Straightforward calculations show that

$$
\begin{gathered}
\frac{\partial w(t, s)}{\partial t}=A(t) w(t, s), \quad s \leq t, t \neq t_{k}, k=1,2, \ldots, \\
w(s, s)=E, \\
w\left(t_{k}+0, s\right)=\left(E+B_{k}\right) w\left(t_{k}, s\right), \quad s<t_{k}, k=1,2, \ldots, \\
w(t, s) w\left(s, t_{0}\right)=w\left(t, t_{0}\right), \quad t_{0}<s<t .
\end{gathered}
$$


Conditions (A) are met if the following hold.

$\left(\mathrm{A}_{1}\right) 0 \leq t_{0}<t_{1}<t_{2}<\cdots<t_{k}<\cdots$, and $\lim _{k \rightarrow \infty} t_{k}=\infty$.

$\left(\mathrm{A}_{2}\right)$ The function $f: T \times \mathbb{R}^{n} \rightarrow \mathbb{R}^{n}$ is continuous, and has a continuous partial derivative $f_{k}$ in $\left(t_{k-1}, t\right) \times \mathbb{R}^{n}, k=1,2, \ldots$ and $f(t, 0)=0$.

$\left(\mathrm{A}_{3}\right)$ For any $x \in \mathbb{R}^{n}$ and any $k=1,2, \ldots$ the functions $f$ and $f_{x}$ have finite limits as $(t, y) \rightarrow\left(t_{k}, x\right) t>t_{k}$.

$\left(\mathrm{A}_{4}\right)$ The function $I_{k}: \mathbb{R}^{n} \rightarrow \mathbb{R}^{n}, k=1,2, \ldots$ are continuous differentiable in $\mathbb{R}^{n}$ and $I_{k}(0)=0, k=1,2, \ldots$.

$\left(\mathrm{A}_{5}\right)$ The solution $x\left(t, t_{0}, x_{0}\right)$ of system (1.1) which satisfies the initial condition $x\left(t_{0}+0, t_{0}, x_{0}\right)=x_{0}$ is defined in the interval $\left(t_{0}, \infty\right)$.

Condition (B) is met if the following holds.

(B) The matrix $A(t)$ is piecwise continuous in $t$ with points of discontinuity of the first kind $t=t_{k}, k=1,2, \ldots$ at which it is continuous from the left.

The following definitions will be needed in the sequel.

DEFINITION 1.1 (see [4]). The zero solution of (1.1) is said to be uniformly stable if for every $\epsilon>0, t_{0} \in \mathbb{R}^{n}, t_{0} \geq 0$, such that

$$
\left\|x_{0}\right\|<\delta \text { implies }\left\|x\left(t, t_{0}, x_{0}\right)\right\|<\epsilon, t \geq t_{0} .
$$

DEFINITION 1.2 (see [4]). The zero solution of (1.1) is said to be asymptotically in variation if for $t \geq t_{0} \geq 0$, there exists $M>0$ such that

$$
\int_{t_{0}}^{t}\|\psi(t, s)\| d s \leq M, \quad \sum_{t_{0} \leq t_{k}<t}\left\|\psi\left(t, t_{k}+0\right)\right\| \leq M .
$$

The following definitions are somewhat new and related with that of [2, 4].

DEFINITION 1.3. The zero solution of system (1.1) is said to be uniformly eventually Lipschitz stable if for $\epsilon>0$, there exist $M>0, \delta(\epsilon)>0$, and $\tau(\epsilon)>0$ such that $\left\|x_{0}\right\| \leq \delta, x_{0} \in \mathbb{R}^{n}$, implies $\left\|x\left(t, t_{0}, x_{0}\right)\right\| \leq M\left\|x_{0}\right\|, t \geq t_{0} \geq \tau(\epsilon)$.

Any eventual Lipschitz stability notions can be similarly defined.

In the case of globally eventually Lipschitz stable, $\delta$ is allowed to be $\infty$.

DEFINITION 1.4. The zero solution of system (1.1) is said to be uniformly eventually stable if for $\epsilon>0$, there exist $M>0, \delta(\epsilon)>0$, and $\tau(\epsilon)>0$, such that for

$$
\left\|x_{0}\right\| \leq \delta \Longrightarrow\left\|x\left(t, t_{0}, x_{0}\right)\right\| \leq \epsilon, \quad t \geq t_{0} \geq \tau(\epsilon), x_{0} \in \mathbb{R}^{n} .
$$

DEFINITION 1.5. The zero solution of (1.1) is said to be uniformly eventually asymptotically stable if it is uniformly eventually stable, and for $\epsilon>0$, there exist $\delta(\epsilon)>0$, and $T(\epsilon)>0$ such that for $x_{0} \in \mathbb{R}^{n}$

$$
\left\|x_{0}\right\| \leq \delta \longrightarrow\left\|x\left(t, t_{0}, x_{0}\right)\right\| \leq \epsilon, \quad t \geq t_{0}+T(\epsilon), t_{0} \geq \tau(\epsilon) .
$$

Any eventually stability notions can be similarly defined.

REMARK 1.6. For Definitions 1.3 and 1.4, if the zero solution of (1.1) is uniformly eventually Lipschitz stable, then it is uniformly Lipschitz stable of [4] and is uniformly eventually stable. 
Now, we state the following result without its proof.

THEOREM 1.7 (see [4]). Let condition (B) be satisfied and let $w$ be the fundamental matrix solution of (1.6). Moreover, let $k, h: J \rightarrow(0, \infty)$ be piecwise continuous functions and exist with points of discontinuity $t=t_{k}, k=1,2, \ldots$ at which they are continuous from the left and such that

$$
\begin{gathered}
\int_{t_{0}}^{t} h(s)\|w(t, s)\| d s \leq k(t), \quad t \geq t_{0} \geq \tau(\epsilon), t \neq t_{k}, k=1,2, \ldots, \\
k(t) \exp \left(-\int_{t_{0}}^{t} \frac{h(s)}{k(s)} d s\right) \leq N, \quad t>t^{*} \geq 0, t \neq t_{k}, k=1,2, \ldots,
\end{gathered}
$$

where $N>0$ is constant. Then the zero solution of (1.6) is uniformly Lipschitz stable.

2. Uniform eventual stability. In this section, we discuss the notion of eventual stability of impulsive systems of differential equations (1.6).

THEOREM 2.1. Let the hypothesis of Theorem 1.7 be satisfied, then the zero solution of (1.6) is uniformly eventually stable.

Proof. From Theorem 1.7, the zero solution of (1.6) is uniformly Lipschitz stable, that is, for $\epsilon>0$, there exist $M>1$ and $\delta>0$ such that

$$
\left\|x_{0}\right\| \leq \delta, \quad x_{0} \in \mathbb{R}^{n} \text { implies }\left\|x\left(t, t_{0}, x_{0}\right)\right\| \leq M\left\|x_{0}\right\|, t \geq t_{0}>0 .
$$

Now, if we choose $\delta_{1}=\min [\delta, \epsilon / 2 M]$, and $\tau(\epsilon) \geq 0$, then we get $\left\|x_{0}\right\| \leq \delta$, for $x_{0} \in \mathbb{R}^{n}$, implies

$$
\left\|x\left(t, t_{0}, x_{0}\right)\right\| \leq M \delta=M \frac{\epsilon}{2 M}=\frac{\epsilon}{2}<\epsilon, \text { for } t \geq t_{0} \geq \tau(\epsilon)>0 .
$$

Hence, the zero solution of (1.6) is uniformly eventually stable.

Consider the scalar impulsive differential equation

$$
u^{\prime}=g(t, u), \quad t \neq t_{k} ; \quad u\left(t_{k}+0\right)=G_{k}\left(u\left(t_{k}\right)\right) ; \quad u\left(t_{0}+0\right)=u_{0} \geq 0,
$$

where $g(t, u) \in C\left[J \times \mathbb{R}^{+}, \mathbb{R}^{+}\right]$, and $g(t, 0)=0$.

THEOREM 2.2. Let conditions $\left(A_{1}\right)-\left(A_{5}\right)$ be satisfied and let there exist functions $g(t, u) \in C\left[J \times \mathbb{R}^{+}, \mathbb{R}^{+}\right], g(t, 0)=0$, and $G_{k}:\left[0, \rho_{0}\right) \rightarrow[0, \rho), G_{k} \in k$ such that for $(t, x) \in J \times S(\rho)$ and for any $h>0$, is small enough, the following inequalities

$$
\begin{aligned}
\|x+h f(t, x)\| & \leq\|x\|+h g(t,\|x\|)+\epsilon(h), \\
\left\|x+I_{k}(x)\right\| & \leq G_{k}(\|x\|), \quad k=1,2, \ldots,
\end{aligned}
$$

are valid, where $\epsilon(h) / h \rightarrow 0$ as $h \rightarrow 0$. If the zero solution of (2.3) is uniformly eventually stable, then so is the zero solution of (1.1).

Proof. From the assumption, the zero solution of (2.3) is uniformly eventually stable, it follows that there exist $\delta(\epsilon)>0$ and $\tau(\epsilon)>0$, for all $\epsilon>0$ such that

$$
u\left(t, t_{0}, u_{0}\right)<\epsilon, \text { for } 0 \leq u_{0}<\delta, t \geq t_{0} \geq \tau(\epsilon),
$$

where $u\left(t, t_{0}, u_{0}\right)$ is any solution of (2.3) for which $u\left(t_{0}+0, t_{0}, u_{0}\right)=u_{0}$. 
Now, we prove that

$$
\left\|x\left(t, t_{0}, x_{0}\right)\right\|<\epsilon, \quad t \geq t_{0}>\tau(\epsilon)
$$

whenever $\left\|x_{0}\right\| \leq \delta$. Suppose that this is not true, then for a solution $x(t)=x\left(t, t_{0}, x_{0}\right)$ of (1.1), $\left\|x_{0}\right\| \leq \delta$, there exists $t_{1} \in\left(t_{k}, t_{k+1}\right)$ for some positive $k$ such that

$$
\left\|x\left(t_{1}\right)\right\|>\epsilon, \quad\|x(t)\| \leq \epsilon, \quad t_{0} \leq t \leq t_{k} .
$$

From (2.5), it follows that

$$
\left\|x\left(t_{K}+0\right)\right\|=\left\|x\left(t_{k}\right)+I_{k} x\left(t_{k}\right)\right\| \leq G_{k}\left(\left\|x\left(t_{k}\right)\right\|\right) \leq G_{k}(\epsilon) .
$$

Let $\rho_{1}=\min \left(\rho, \rho_{0}\right)$, we get

$$
\left\|x\left(t_{k}+0\right)\right\| \leq G_{k}(\epsilon) \leq \rho .
$$

Hence there exists $t_{2}, t_{k}<t_{2} \leq t_{1}$, such that

$$
\epsilon<\left\|x\left(t_{2}\right)\right\|<\rho, \quad\|x(t)\|<\rho, \quad t_{0}<t \leq t_{2} .
$$

Let $V(t)=\|x(t)\|$. From (2.5), it follows that for $t \in\left(t_{0}, t_{2}\right], t \neq t_{j}, j=1,2, \ldots, k$ the following inequalities hold:

$$
\begin{aligned}
V^{\prime}(t) & =\operatorname{Lim}_{h \rightarrow 0}\left(\frac{1}{h}\right)[\|x(t+h)\|-\|x(t)\|] \\
& \leq \operatorname{Lim}_{h \rightarrow 0}\left(\frac{1}{h}\right)[\|x(t+h)\|+h g(t,\|x(t)\|)+\epsilon(h)-\|x(t)+h f(t, x(t))\|] \\
& \leq g(t,\|x(t)\|)+\operatorname{Lim}_{h \rightarrow 0}\left(\frac{\epsilon(h)}{h}\right)+\operatorname{Lim}_{h \rightarrow 0}\left\|\left(\frac{1}{h}\right)[x(t+h)-x(t)]-f(t, x)\right\| \\
& =g(t,\|x(t)\|)=g(t, V(t)) .
\end{aligned}
$$

From (2.5), we get that for $j=1,2, \ldots, k$, the inequalities

$$
V\left(t_{j}+0\right)=\left\|x\left(t_{j}+0\right)\right\|=\left\|x\left(t_{j}\right)+\tau_{j}\left(x_{j}\right)\right\| \leq G_{j}\left(\left\|x\left(t_{j}\right)\right\|\right)
$$

hold, hence

$$
V\left(t_{j}+0\right) \leq G_{j}\left(m\left(t_{j}\right)\right), \quad j=1,2, \ldots, m k
$$

Moreover,

$$
V\left(t_{0}+0\right)=\left\|x\left(t_{0}+0\right)\right\|=\left\|x_{0}\right\|=V_{0} .
$$

Applying the comparison [5, Theorem 1.4.3], yields

$$
\|x(t)\|=V(t) \leq u(T), \quad t<t_{1} \leq t_{2} .
$$

From (2.6), (2.11), and (2.16), it follows that

$$
\epsilon<\left\|x\left(t_{2}\right)\right\|=V\left(t_{2}\right) \leq u(t)<\epsilon .
$$


This is a contradiction, thus $\left\|x\left(t, t_{0}, x_{0}\right)\right\|<\epsilon$, for $t \geq t_{0} \geq \tau(\epsilon)$, whenever $\left\|x_{0}\right\|<$ $\delta$. Hence the zero solution of (1.1) is uniformly eventually stable, and the proof is completed.

THEOREM 2.3. Let the hypothesis of Theorem 2.2 be satisfied except the condition (2.5) is replaced by

$$
[x, f(t, x)] \leq g(t,\|x(t)\|),
$$

where

$$
[x, y]_{+}=\operatorname{Lim}_{h \rightarrow 0^{+}} \sup \left(\frac{1}{h}\right)(\|x+h y\|-\|x\|), \quad x, y \in \mathbb{R}^{n} .
$$

Then the zero solution of (1.1) is uniformly eventually stable.

Proof. The proof is very similar to the proof of Theorem 2.2, but from (2.18), we obtain for $t \in\left(t_{0}, t_{2}\right], t \neq t_{j}, j=1,2, \ldots, k$, the following inequalities are satisfied

$$
\begin{aligned}
D^{+} V(t)= & \operatorname{Lim}_{h \rightarrow 0^{+}} \sup \left(\frac{1}{h}\right)[V(t+h)-V(t)] \\
= & \operatorname{Lim}_{h \rightarrow 0^{+}} \sup \left(\frac{1}{h}\right)[\|x(t+h)\|-\|x(t)\|] \\
\leq & \operatorname{Lim}_{h \rightarrow 0^{+}} \sup \left\|\left(\frac{1}{h}\right)[x(t+h)-x(t)]-f(t, x)\right\| \\
& +\operatorname{Lim}_{h \rightarrow 0^{+}} \sup \left(\frac{1}{h}\right)[\|x(t)+h f(t, x)\|-\|x(t)\|] \\
= & {[x(t), f(t, x)] \leq g(t, V(t)) . }
\end{aligned}
$$

The rest of the proof is in the same line of the proof of Theorem 2.2, so it is omitted.

3. Uniform eventual Lipschitz stability. In this section, we discuss the notion of uniform eventual Lipschitz stability of the linear system (1.6).

THEOREM 3.1. Let the zero solution of (1.8) be uniformly Lipschitz stable in variation for the linear system (1.6). The following statements are equivalent.

(i) The zero solution of (1.6) is globally uniformly eventually Lipschitz stable in variation.

(ii) The zero solution of (1.6) is uniformly eventually Lipschitz stable in variation.

(iii) The zero solution of (1.6) is globally uniformly eventually Lipschitz stable.

(iv) The zero solution of (1.6) is uniformly eventually Lipschitz stable.

(v) The zero solution of (1.6) is uniformly eventually stable.

Proof. (i) $\Rightarrow$ (ii). This follows directly from Definition 1.3.

(ii) $\Rightarrow$ (iii). This follows from (1.9), the definition of the fundamental matrix $w(t, s)$ of (1.6) which is independent of $x_{0}$, thus from our assumption, we get

$$
\left\|x\left(t, t_{0}, x_{0}\right)\right\|=\left\|w\left(t, t_{0}\right) x_{0}\right\| \leq\left\|w\left(t, t_{0}\right)\right\|\left\|x_{0}\right\| \leq M\left\|x_{0}\right\|,
$$

for $\left\|x_{0}\right\| \in \mathbb{R}^{n}, t \geq t_{0} \geq \tau(\epsilon)$, and $M>1$.

Then the zero solution of (1.6) is globally uniformly eventually Lipschitz stable. 
(iii) $\Rightarrow$ (iv). This follows immediately from Definition 1.3.

(iv) $\Rightarrow$ (v). Let the zero solution of (1.6) be uniformly eventually Lipschitz stable, then for $\epsilon>0$, there exists $M>0, \delta(\epsilon)>0$, and $\tau(\epsilon)>0$, such that

$$
\left\|x\left(t, t_{0}, x_{0}\right)\right\| \leq M\left\|x_{0}\right\|, \quad t \geq t_{0} \geq \tau(\epsilon)
$$

whenever $\left\|x_{0}\right\| \delta$.

Now, if we choose $\delta_{1}=\min (\delta, \epsilon / 2 M)$, then for $\left\|x_{0}\right\| \leq \delta$, we have

$$
\left\|x\left(t, t_{0}, x_{0}\right)\right\| \leq M\left\|x_{0}\right\| \leq M \delta_{1}<\epsilon, \quad t \geq t_{0} \geq \tau(\epsilon) .
$$

Hence the zero solution of (1.6) is uniformly eventually stable.

(v) $\Rightarrow$ (i). Let the zero solution of (1.6) be uniformly eventually stable, then from our assumption, we get

$$
\left\|w\left(t, t_{0}\right)\right\| \leq M, \quad M>0
$$

where $w\left(t, t_{0}\right)$ is the fundamental matrix solution of (1.6). Hence (i) is obtained, and the proof is completed.

THEOREM 3.2. Let the hypothesis of Theorem 2.2 be satisfied, and if the zero solution of (2.3) is uniformly eventually Lipschitz stable, then so is the zero solution of (1.1).

Proof. From the assumption that the zero solution of (2.3) is uniformly eventually Lipschitz stable, it follows that there exist $M>1, \tau(\epsilon)>0$, and $\delta(\epsilon)>0$ such that for $\epsilon>0$

$$
u\left(t, t_{0}, u_{0}\right) \leq M u_{0}, \quad \text { for } 0 \leq u_{0}<\delta, t \geq t_{0} \geq \tau(\epsilon)
$$

where $u\left(t, t_{0}, u_{0}\right)$ is any solution of (2.3) for which $u\left(t_{0}+0, t_{0}, u_{0}\right)=u_{0}$.

Now, we prove that

$$
\left\|x\left(t, t_{0}, x_{0}\right)\right\| \leq M\left\|x_{0}\right\|, \quad t \geq t_{0} \geq \tau(\epsilon), \text { for }\left\|x_{0}\right\|<\delta .
$$

Suppose that this is not true, then for a solution $x(t)=x\left(t, t_{0}, x_{0}\right)$ of $(1.1),\left\|x_{0}\right\|<$ $\delta$ there exists $t_{1} \in\left(t_{k}, t_{k+1}\right)$ for some positive $k$ such that $\left\|x\left(t_{1}\right)\right\|>M\left\|x_{0}\right\|$, and $\|x(t)\| \leq M\left\|x_{0}\right\|, t_{0} \leq t \leq t_{k}$. From (2.5), it follows that

$$
\left\|x\left(t_{k}+0\right)\right\|=\left\|x\left(t_{k}\right)+I_{k} x\left(t_{k}\right)\right\| \leq G_{k}\left(\left\|x\left(t_{k}\right)\right\|\right) \leq G_{k}\left(\left\|x_{0}\right\|\right)<G_{k}(M \delta) .
$$

Let $\rho_{1}=\min \left(\rho, \rho_{0}\right)$, we get

$$
\left\|x\left(t_{k}+0\right)\right\| \leq G_{k}(M \delta)<G\left(\rho_{1}\right) \leq \rho .
$$

Hence, there exists $t_{2}, t_{k}<t_{2} \leq t_{1}$, such that

$$
M\left\|x_{0}\right\|<\left\|x\left(t_{2}\right)\right\|<\rho, \quad\|x(t)\|<\rho, \quad t_{0}<t \leq t_{2} .
$$


Let $V(t)=\|x(t)\|$, and $u_{0}=\left\|x_{0}\right\|$. From (2.5), the following inequalities are satisfied

$$
\begin{aligned}
V^{\prime}(t) & =\operatorname{Limsup}_{h \rightarrow 0}\left(\frac{1}{h}\right)[\|x(t+h)\|-\|x(t)\|] \\
& \leq \operatorname{Lim}_{h \rightarrow 0} \sup \left(\frac{1}{h}\right)[\|x(t+h)\|+h g(t,\|x(t)\|)+\epsilon(h)-\|x(t)+h f(t, x(t))\|] \\
& \leq g(t,\|x(t)\|)+\operatorname{Lim}_{h \rightarrow 0}\left(\frac{\epsilon(h)}{h}\right)+\operatorname{Lim}_{h \rightarrow 0}\left\|\frac{1}{h}[x(t+h)-x(t)]-f(t, x)\right\| \\
& =g(t,\|x(t)\|)=g(t, V(t)) .
\end{aligned}
$$

From (2.5), for $j=1,2, \ldots, k$, the inequalities

$$
V\left(t_{j}+0\right)=\left\|x\left(t_{j}+0\right)\right\|=\left\|x\left(t_{j}\right)+I_{j}\left(x\left(t_{j}\right)\right)\right\| \leq G_{j}(\|x(t)\|)
$$

hold, hence

$$
V\left(t_{j}+0\right) \leq G_{j}\left(V\left(t_{j}\right)\right), \quad j=1,2, \ldots, k .
$$

Moreover,

$$
V\left(t_{0}+0\right)=\left\|x\left(t_{0}+0\right)\right\|=\left\|x_{0}\right\|=V_{0},
$$

applying the comparison [5, Theorem 1.4.3], we get

$$
\|x(t)\|=V(t) \leq u\left(t, t_{0}, u_{0}\right), \quad t_{0}<t<t_{2} .
$$

From (3.5), (3.9), and (3.14), it follows that

$$
M\left\|x_{0}\right\|<\left\|x\left(t_{2}\right)\right\|=V\left(t_{2}\right) \leq u\left(t, t_{0}, u_{0}\right) \leq M u_{0}=M\left\|x_{0}\right\| .
$$

This is a contradiction, thus $\left\|x\left(t, t_{0}, x_{0}\right)\right\| \leq M\left\|x_{0}\right\|$, for $t \geq t_{0} \geq \tau(\epsilon)$, whenever $\left\|x_{0}\right\|<$ $\delta$. Hence the zero solution of (1.1) is uniformly eventually Lipschitz stable, and the proof is completed.

THEOREM 3.3. Let conditions $\left(A_{1}\right)-\left(A_{5}\right)$ be satisfied and let the zero solution of (1.1) be eventually asymptotically stable in variation. Then the zero solution of (1.1) is uniformly eventually Lipschitz stable.

Proof. Let $\psi\left(t, t_{0}\right)$ be the fundamental matrix solution of (1.4). From [4, Corollary 1], it follows that

$$
\left\|\psi\left(t, t_{0}\right)\right\| \leq k_{1}, \quad \text { for } t \geq t_{0} \geq \tau(\epsilon) \geq 0
$$

where $k_{1}>0$ is constant. From our assumption, it follows that

$$
\int_{t_{0}}^{t}\|\psi(t, s)\| \leq k_{2}, \quad \sum_{t_{0}<t_{k}<t}\left\|\psi\left(t, t_{k}+0\right)\right\| \leq k_{2},
$$

for $t \geq t_{0} \geq \tau(\epsilon)>0, k_{2}>0$ is constant. Let $k=\max \left[k_{1}, k_{2}\right]$, since $f(t, 0)=0$, and $I_{k}=0, k=1,2, \ldots$ It follows that for $\epsilon=1 / 2 k$, there exists $\delta>0$, such that $f(t, x)=$ 
$f_{x}(t, 0) x+h(t, x)$, and $I_{k}(x)=I_{k}^{\prime}(x) x+h_{k}(x)$, for $\left\|x_{0}\right\|<\delta$, where $\|h(t, x)\|<\epsilon\|x\|$ and $\left\|h_{k}\right\|<\epsilon\|x\|, k=1,2, \ldots$.

By applying the variation of constants formula (see [6, page 266]), we obtain

$$
\begin{aligned}
\left\|x\left(t, t_{0}, x_{0}\right)\right\| \leq & \left\|\psi\left(t, t_{0}+0\right) x_{0}\right\|+\int_{t_{0}}^{t}\|\psi(t, s)\|\left\|h\left(s, x\left(s, t_{0}, x_{0}\right)\right)\right\| d s \\
= & \sum_{t_{0}<t_{k}<t}\left\|\psi\left(t, t_{0}+0\right)\right\|\left\|h_{k}\left(x\left(t_{k}, t_{0}, x_{0}\right)\right)\right\| \\
\leq & k\left\|x_{0}\right\|+\epsilon \int_{t_{0}}^{t}\|\psi(t, s)\|\left\|x\left(s, t_{0}, x_{0}\right)\right\| d s \\
& +\epsilon \sum_{t_{0}<t_{k}<t}\left\|\psi\left(t, t_{0}+0\right)\right\|\left\|x\left(t_{k}, t_{0}, x_{0}\right)\right\| \\
\leq & k\left\|x_{0}\right\|+2 \epsilon k \sup _{t \leq s \leq t}\left\|x\left(s, t_{0}, x_{0}\right)\right\| .
\end{aligned}
$$

Hence,

$$
\left\|x\left(t, t_{0}, x_{0}\right)\right\| \leq \frac{k}{1-2 \epsilon k}\left\|x_{0}\right\|=M\left\|x_{0}\right\|, \quad t \geq t_{0} \geq \tau(\epsilon)>0,
$$

and the result is immediate.

4. Examples. Now, we illustrate the results obtained by some examples.

EXAMPLE 4.1. Consider the linear impulsive system (1.6) for which conditions $\left(\mathrm{A}_{1}\right)$ $\left(\mathrm{A}_{5}\right)$ and (B) hold. If, moreover, the following conditions hold:

(a) $\operatorname{Lim}_{t \rightarrow \infty} \sup _{t_{0} \leq s \leq t}\left(\int_{t_{0}}^{t} u(A(s)) d s\right)<\infty$.

(b) $\left\|E+B_{k}\right\| \leq d_{k}, k=1,2, \ldots$.

(c) $\prod_{k=1}^{\infty} d_{k}<\infty$.

Then the zero solution of the scalar impulsive differential equation

$$
u^{\prime}=\mu(A(t)) u, \quad t \neq t_{k} ;\left.\quad \Delta u\right|_{t=t_{k}}=\left(d_{k}-1\right) u ; \quad u\left(t_{0}+0\right)=u_{0}>0,
$$

is uniformly eventually stable. Then the condition of Theorem 2.1 holds, it follows that the zero solution of (1.6) is uniformly eventually stable.

EXAMPLE 4.2. Consider the impulsive system of differential equation (1.1). Let the conditions $\left(\mathrm{A}_{1}\right)-\left(\mathrm{A}_{5}\right)$ hold as the following conditions:

(a) $[x, f(x)] \leq \rho(t) F(\|x\|)$, for $(t, x) \in J \times s(\rho)$, where $\rho \in C\left[\mathbb{R}^{+}, \mathbb{R}^{+}\right]$, and $F \in K$.

(b) $\left\|x+I_{k}(x)\right\| \leq G_{k}(\|x\|)$, for $x \in s(\rho) ; k=1,2, \ldots$ where $G_{k}:\left[0, \rho_{0}\right) \rightarrow[0, \rho)$ and $G_{k} \in K, k=1,2, \ldots$.

(c) For any $\sigma \in\left(0, \rho_{0}\right)$, the following inequality holds

$$
\int_{t_{k}}^{t_{k+1}} \rho(s) d s+\int_{\sigma}^{G_{k}(\sigma)} \frac{d s}{F(s)} \leq 0, \quad k=1,2, \ldots
$$

Then the zero solution of scalar impulsive differential equation

$$
u^{\prime}=\rho(t) F(u), \quad t \neq t_{k}, \quad \Delta u=G_{k}\left(u\left(t_{k}\right)\right)-u\left(t_{k}\right), \quad u\left(t_{0}+0\right)=u_{0} \leq 0,
$$

is uniformly eventually Lipschitz stable, thus the conditions of Theorem 3.2 hold, it follows that the zero solution of system (1.1) is uniformly eventually Lipschitz stable. 
ACKNOWLEDGEMENT. The author would like to thank the referees for their valuable comments and suggestions on the manuscript.

\section{REFERENCES}

[1] D. D. Baĭnov and P. S. Simeonov, Systems with Impulsive Effect Stability, Theory and Applications, Ellis Horwood Series in Mathematics and its Applications, Ellis Horwood, Chichester, 1989. Zbl 683.34032.

[2] F. M. Dannan and S. Elaydi, Lipschitz stability of nonlinear systems of differential equations, J. Math. Anal. Appl. 113 (1986), no. 2, 562-577. MR 87e:34088. Zbl 595.34054.

[3] G. K. Kulev and D. D. Bănov, Lipschitz quasistability of impulsive differential equations, J. Math. Anal. Appl. 172 (1993), no. 1, 24-32. MR 94b:34074. Zbl 772.34042.

[4] _ Lipschitz stability of impulsive systems of differential equations, Dynam. Stability Systems 8 (1993), no. 1, 1-17. MR 94i:34102. Zbl 787.93051.

[5] V. Lakshmikantham, D. D. Bănov, and P. S. Simeonov, Theory of Impulsive Differential Equations, Series in Modern Applied Mathematics, vol. 6, World Scientific, New Jersey, 1989. MR 91m:34013. Zbl 719.34002.

[6] A. M. Samoilenko and N. A. Perestyuk, Differential Equations with Impulsive Effect, Višča Škola, Kiev, 1987 (Russian).

A. A. Soliman: Department of Mathematics, Faculty of Sciences, Benha University, BENHA 13518, KALUBIA, EGYPT

Current address: Department of Mathematics, Faculty of Teachers, Al-Jouf, SKaka, P.O. BOX 269, SAUDI ARABIA

E-mail address: a_a_soliman@hotmai 1 .com 


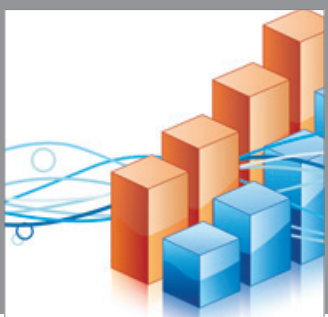

Advances in

Operations Research

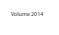

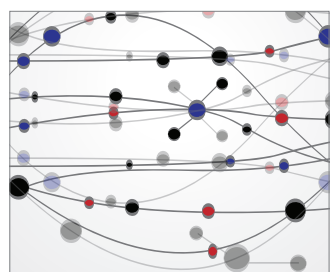

\section{The Scientific} World Journal
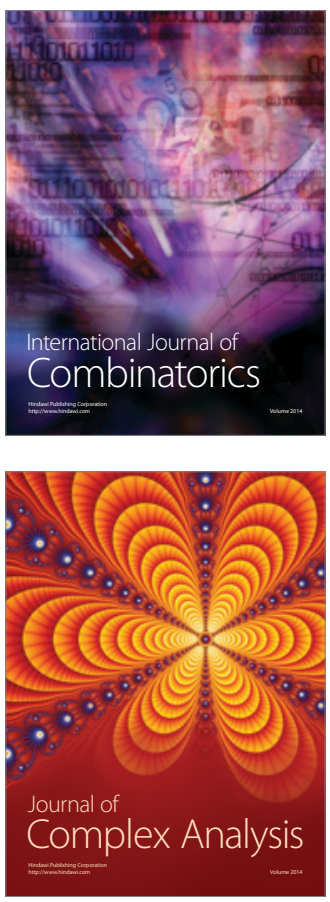

International Journal of

Mathematics and

Mathematical

Sciences
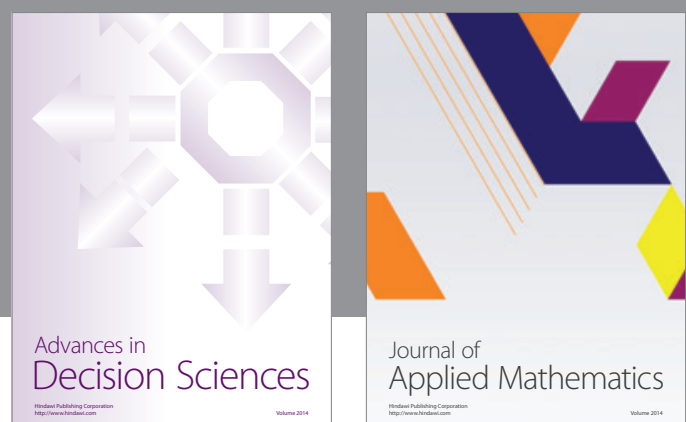

Journal of

Applied Mathematics
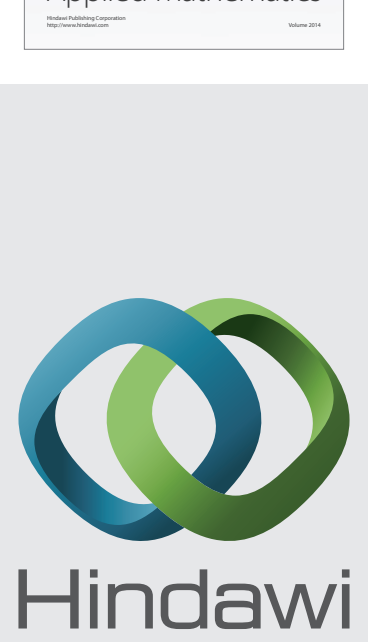

Submit your manuscripts at http://www.hindawi.com
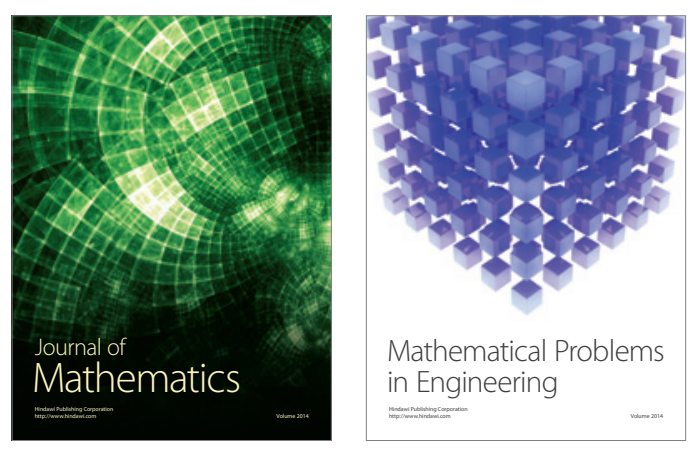

Mathematical Problems in Engineering
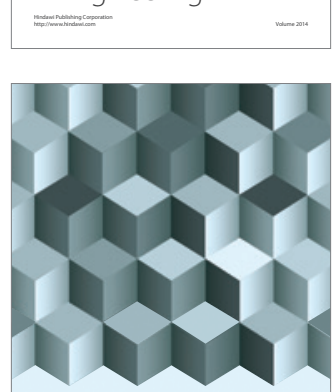

Journal of

Function Spaces
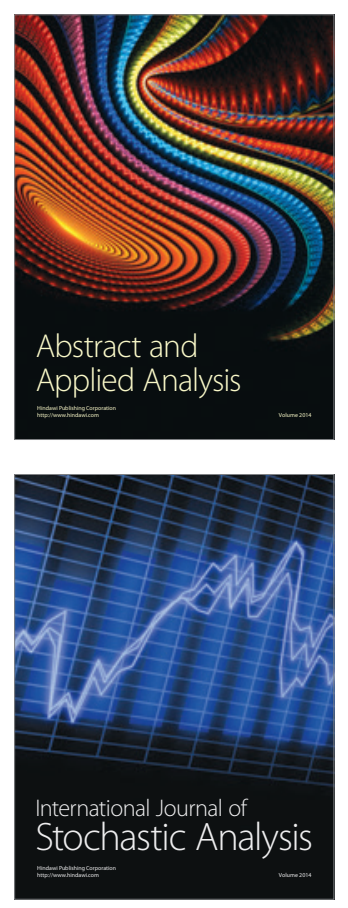

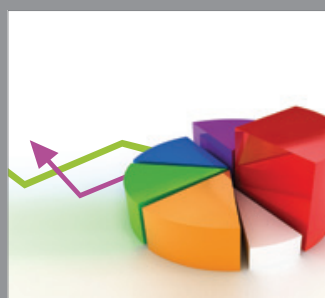

ournal of

Probability and Statistics

Promensencen
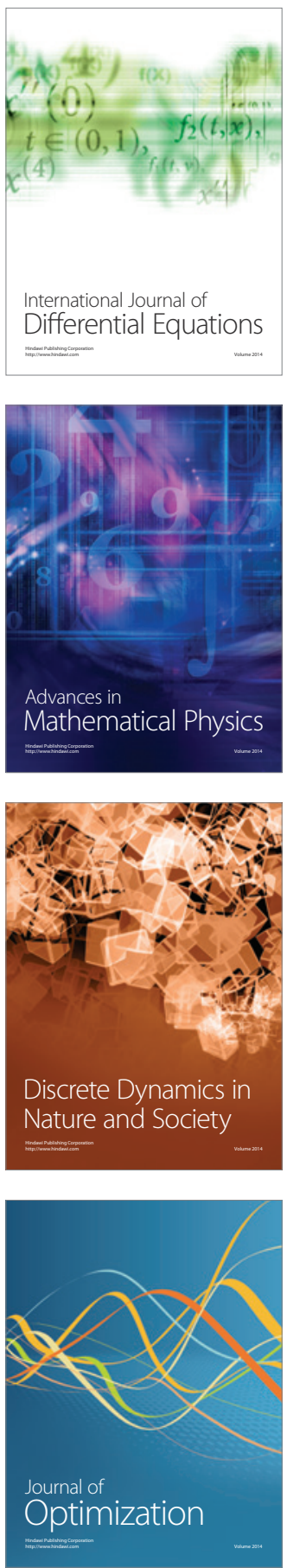